\title{
The effect of hydrogen induced cracking on the integrity of steel components
}

\author{
INDRANIL CHATTORAJ \\ National Metallurgical Laboratory, Jamshedpur 831007 , India
}

\begin{abstract}
The occurrence of hydrogen embrittlement is a much researched phenomenon, known to cause mechanical property degradation and catastrophic failures. The ductility loss brought about by hydrogen ingress is encountered even in unstressed bodies where such cracking is termed hydrogen induced cracking ( $\mathrm{HIC}$ ) and is in phenomenological contrast to catastrophic failures encountered by stressed bodies subjected to hydrogen producing environments. This article will discuss HIC in some detail. This form of cracking is especially detrimental and often observed in oil country tubular goods (OCTG) which are subjected to sour gas. Consequently, the significance of $\mathrm{HIC}$ is most appreciated by oil companies at various stages of oil extraction. transportation and storage. In this article the chemical and metallurgical genesis of $\mathrm{HIC}$, its harmful impact on material and component integrity are discussed. It has been noted that $\mathrm{MnS}$ inclusions are extremely harmful to this form of cracking. Similarly, centreline segregation in the ingot stage and deoxidation practices during steelmaking were found to affect HIC. Some case studies of HIC obtained from literature are presented. The variables affecting the propensity to HIC are provided in brief. Suitable measures to reduce or eliminate $\mathrm{HIC}$ in steels are also discussed.
\end{abstract}

Keywords. Hydrogen-induced cracking; embrittlement, decohesion theory; hydrogen absorption.

\section{Introduction}

Hydrogen-induced cracking ( $\mathrm{HIC}$ ) is a phenomenon widely encountered by components exposed to environments capable of introducing hydrogen into the component material, especially sour environments to which oil and gas installations and transmission systems are exposed. The presence of $\mathrm{H}_{2} \mathrm{~S}$ in such environments catalyse hydrogen entry into steels. HIC can be thought of as one form of hydrogen embrittlement, and is differentiated from the other forms of hydrogen embrittlement phenomena by the appearance, morphology and sites of cracking and by the mode of crack propagation. Hydrogen induced cracking occurs at ambient or near-ambient conditions and in stressed as well as unstressed cornponents. These cracks have a planar appearance, with 
different cracks linked in a stepwise fashion. HIC is often accompanied by surface blisters.

This article deals with the general occurrence of hydrogen entry and hydrogen trapping and with the theories of hydrogen embrittlement, to provide a background of the various kinetic processes causing material degradation by hydrogen ingress. Subsequently hydrogen induced cracking is dealt with by providing a brief introduction of the phenomenon, discussing the variables affecting HIC and providing case studies including our recent experiences with this failure mode. The various preventive measures to counter HIC are also discussed.

\section{Hydrogen entry}

The hydrogen evolution reaction was characterized by McBreen \& Genshaw (1967). The evolution of hydrogen occurs frequently due to cathodic reduction of $\mathrm{H}^{+}$ions in aqueous corroding solutions, or hydrogen may be absorbed due to the use of high pressure hydrogen gas in the environment. In addition, the material itself may have hydrogen retained from the process of ore reduction (steelmaking for example). Hydrogen either absorbed from the environment or present in the material can lead to unpredictable catastrophic failure. This is evident in the various failures in the oil and gas industry, aircraft and automotive industries.

The problem of hydrogen embrittlement of any material involves various mechanisms which finally lead to embrittlement. The steps can be broadly classified as follows.

(1) Hydrogen adsorption at the metal/alloy surface.

(2) Hydrogen evolution at the metal/alloy surface.

(3) Absorption of some of the adsorbed hydrogen into the bulk of the metal.

(4) Transport of absorbed hydrogen to normal lattice sites as well as potential sites for cracking, under the influence of a naturally existing or externally applied chemical potential gradient.

(5) Accumulation of hydrogen at the crack sites leading to catastrophic failure.

Various schematics have been proposed for the reaction sequence mentioned above. McBreen \& Genshaw (1967) proposed a simple scheme for the evolution and entry reaction and over the years it has served as a satisfactory basis for iron and steels and is included here in figure 1 . The overall reaction of hydrogen evolution in solutions are as follows,

$$
\begin{aligned}
& 2 \mathrm{H}_{3} \mathrm{O}^{+}+2 e^{-}=\mathrm{H}_{2}+2 \mathrm{H}_{2} \mathrm{O} \quad \text { (in acid solutions), } \\
& 2 \mathrm{H}_{2} \mathrm{O}+2 e^{-}=\mathrm{H}_{2}+2 \mathrm{OH}^{-} \quad \text { (in alkaline solutions). }
\end{aligned}
$$

The overall reaction can proceed by two mechanisms namely:

(a) The recombination mechanism which involves chemical recombination of adsorbed hydrogen atoms as follows

$$
\begin{aligned}
& \text { i. } \mathrm{M}+\mathrm{H}^{+}+\mathrm{e}^{-}=\mathrm{MH}_{\mathrm{ads}}, \\
& \text { ii. } \mathrm{MH} \mathrm{ads}_{\mathrm{ads}}+\mathrm{MH}_{\mathrm{ads}}=\mathrm{H}_{2}+2 \mathrm{M} \text {. }
\end{aligned}
$$

(b) The electrochemical desorption mechanism which involves the following 


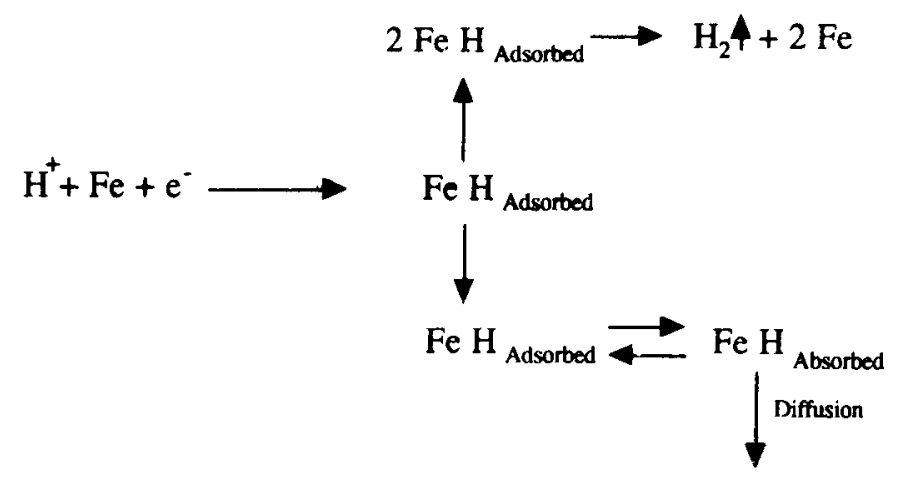

Hydrogen flux through metal

Figure 1. Hydrogen evolution and entry scheme (according to McBreen \& Genshaw 1967).

electrochemical steps,

$$
\begin{aligned}
& \text { i. } \mathbf{M}+\mathrm{H}^{+}+e^{-}=\mathrm{MH}_{\mathrm{ads}}, \quad \text { (as above) } \\
& \text { ii. } \mathrm{MH}_{\mathrm{ads}}+\mathrm{H}^{+}+e^{-}=\mathrm{H}_{2}+\mathrm{M} \text {. }
\end{aligned}
$$

As a result of the need for multiple steps, it is obvious that one of the reaction steps can become rate controlling in the overall reaction path.

\section{Hydrogen trapping}

Darken \& Smith (1949) were the first to observe that hydrogen diffusion in steels was affected by imperfections in the lattice. The first formalism for trapping in steels was developed by McNabb \& Foster (1972). They suggested a trapping and a detrapping parameter and they came up with a mathematical formulation based on the assumption of local equilibrium between trapped and normal lattice sites. Their formalism considered only the reversible traps; irreversible traps were considered not to influence the diffusion as they represented sinks with hydrogen essentially removed from the permeation system. The works of McNabb \& Foster (1972), and Oriani \& Josephic (1974) considered a single population of reversible traps. Pressouyre \& Bernstein (1978) characterized the trapping behaviour of different kinds of traps, both reversible and irreversible, by controlling the type and number of traps and by observing their effect on permeation transient. Johnson et al (1979) analysed saturable and non-saturable traps by considering relationships between the trapped hydrogen concentration and the lattice hydrogen content and redefining effective hydrogen diffusivity in terms of these relationships. According to their theoreticai development, one can predict an increase in apparent hydrogen diffusivity with lattice hydrogen content when saturable traps are present, while the reverse is true for non-saturable traps. To summarize, we can consider traps as either reversible or irreversible depending on the depth of the potential well they present to hydrogen diffusion. Trapping manifests itself by changing apparent diffusivity values. The amount and direction of such a change from perfect lattice diffusion depends on the amount and type of traps present in the lattice. Simultaneous creation of traps and their 
influence on hydrogen ingress, also known as dynamic trapping, occurs during straining of hydrogen charged specimen.

This brief introduction to hydrogen trapping was necessary, since the trapping of hydrogen at $\mathrm{MnS}$ inclusions is the factor most responsible for HIC. The trapped hydrogen causes decohesion of the matrix-inclusion interface, either by pressurization or by reducing the interatomic bond strength. The net effect is the generation of delaminations and cracks near inclusions.

\section{Hydrogen embrittlement theories}

Although many theories have been proposed to explain the hydrogen embrittlement phenomenon, two have gained substantial popularity.

\subsection{Decohesion theory}

This theory was developed by Troiano and others (Troiano 1960; Mortlet et al 1958; Johnson \& Troiano 1957) to explain the synergistic effects of hydrogen on material degradation. Troiano refuted the pressure theory and various surface adsorption theories for embrittlement, as these would predict an initial loss in ductility, in variance with experimental observations. Troiano and co-workers (Troiano 1960; Mortlet et al 1958; Johnson \& Troiano 1957) proposed that the degree of embrittlement would vary as the hydrogen concentration in the region of maximum triaxiality in the lattice. Whereas the other theories were concerned with hydrogen content in cracks and voids, this theory renders hydrogen in voids harmless entities, and only hydrogen in special lattice positions as significant in embrittlement. The role of voids and cracks is to act as stress raisers, so that the region of maximum triaxiality is generally near such imperfections. The disappearance of hydrogen embrittlement at low temperatures can be explained by the decrease of hydrogen diffusivity and at high temperatures by the conflicting effects of stress induced diffusion and increased homogenization. Troiano (1960), made the observation that crack initiation and propagation in HE occurs in three distinct stages: (a) incubation, (b) slow crack growth and (c) catastrophic failure. From fractographic observations, Troiano concluded that the third stage of catastrophic failure was essentially overload failure and there was no effect of hydrogen in this stage. The incubation period according to Troiano was the time required for hydrogen to reach a critical concentration in the region of maximum triaxiality. The slow crack growth in the second stage is essentially the rate of hydrogen diffusion to regions in front of the crack tip, thus implying discontinuous cracking. Troiano suggested a decrease in cohesive strength of the lattice due to presence of hydrogen; this coupled with the observation that hydrogen accumulates at regions of high triaxial stresses near imperfections, explained the embrittlement phenomenon. Troiano's original speculation was that hydrogen atoms in solution in transition metals donate their electrons to the $d$ band of the metallic core, thus increasing the repulsion of metallic cores. This theory predicted that addition of copper to nickel would increase embrittlement initially and then decrease it as the $3 d$ band in nickel is progressively filled; this prediction was substantiated by experimental findings.

Tetelman \& Robertson (1963) observed that crack growth due to hydrogen charging occurred discontinuously. Oriani \& Josephic (1974) demonstrated the existence of 
a threshold stress at a given hydrogen fugacity and conversely a threshold fugacity at a given stress intensity, below which crack growth did not occur in steels. They observed that the threshold values for deuterium were consistently higher than those for hydrogen. This observation can only be reconciled with the decohesion theory; deuterium has a lower molal volume than hydrogen in $x$-iron, thus it is less effective in concentrating at triaxiality sites due to stress induced diffusion. According to Oriani, decohesion due to hydrogen is manifested in high strength steels by an increase in cleavage fracture and in low strength steels by an enhancement of microvoid nucleation. Stress relaxation studies (Oriani \& Josephic 1979) on AISI 1045 steels were explained by suggesting that since hydrogen causes decohesion, it favours microvoid generation over relaxation by dislocation activity. Oriani \& Josephic (1980) also proposed that the reduction of Peierl's stress and the reduction of screw dislocation cross slip in bcc iron are due to reduced cohesive strength in the presence of hydrogen.

Birnbaum's objection to this concept of lowering of cohesive strength is that such a reduction should also manifest itself in the reduction of atomic force constants and elastic constants. In the case of $f c c$ metals a decrease in these constants is indeed observed in the presence of hydrogen. However in group $\mathrm{Vb}$ metals like niobium, even at very high hydrogen charging, there is significant increase in $C_{11}$ and bulk modulus values (Birnbaum \& Mazzolai 1985). The other criticism of this theory is in the appearance of fractures. Decohesion theory predicts that intergranular fracture surface should show the morphology of grain interface whereas actual observations with high resolution microscopes reveal fracture along slip planes near grain boundaries, often crossing over into adjacent grains along slip planes (Robertson et al 1984). In spite of these criticisms, decohesion theory is one of the more popular theories of HE. Calculations based on the embedded atom model and the cluster variation method (Briant \& Messmer 1980) do suggest that hydrogen would indeed cause decohesion, thus vindicating this theory.

\subsection{Internal pressure theory}

A theory of hydrogen embrittlement based on hydrogen accumulation at voids was proposed by Zapffe \& Sims (1941). According to these authors hydrogen atoms accumulate at voids until an equilibrium pressure is attained. When this pressure attains a value close to the elastic strength of the metal, the metal embrittles, de Kazinczy (1954) elaborated on this concept by suggesting that crack growth is facilitated by the energy released by expanding hydrogen gas in cracks. de Kazinczy utilized Griffith's theory of brittle fracture, which states that for a stable crack to grow, the system must undergo a total decrease in the energy content. He suggested that part of the total energy of the material charged with hydrogen is due to the energy content of hydrogen gas in the cracks and voids. On crack propagation, the gas expands releasing energy and thus the energy due to strain required to meet Griffith's criterion is reduced, that is, the strain at which crack propagates is lower. This model however requires that the material have preexisting cracks in it. However Bilby \& Hewitt (1962) and Garofalo et al (1960) proposed that crack initiation could be due to dislocation pile-up under the influence of external stress. Bastien \& Azou (1951) suggested that hydrogen is concentrated in Cottrell atmospheres of dislocations and is transported along with dislocations to the cavities. This dislocation enhanced diffusion of hydrogen would account for the incubation time and temperature effects of embrittlement. 
Bockris \& Subramanyan (1971) developed relationships for the hydrogen fugacity in microcracks and voids as a function of overpotential, in electrochemical charging of hydrogen. Based on theoretical calculations, they predicted which rate controlling mechanisms for hydrogen evolution would cause embrittlement when pressure theory was valid. Beck et al (1965) suggested that blister formation due to high internal pressure of hydrogen in cavities would lead to plastic deformation in the vicinity of blisters. This was supposed to increase the dislocation density near blisters, which would act as crack initiation sites.

There are two criticisms to this theory. Johnson \& Hirth (1976) showed that hydrogen supersaturations obtainable at dislocations were greatly overestimated in literature, and dislocation transport, even if it was effective, could not account for large internal pressure required by this theory. The findings of Hancock \& Johnson (1965), that crack propagation occurs even at very low hydrogen pressure environments, also contradicts the internal pressure mechanism of embrittlement. Such a mechanism might be effective in high fugacity environments but cannot be accepted as a general theory.

\section{Hydrogen-induced cracking (HIC)}

In the previous sections an introduction to the broad area of hydrogen embrittlement was provided. This was done to impress upon the readers that the general effect of hydrogen entry into alloys, especially steels, is a deterioration of their mechanical properties. However the external manifestation of such deterioration can take up various forms depending on the presence or absence of other variables in the system. The aim of this paper is to concentrate on HIC which is a specific type of hydrogen embrittlement, and is often encountered by components exposed to sour environments. The rest of this article will deal with the various parameters enhancing $\mathrm{HIC}$, a few case studies of HIC observed in service, and measures adopted to counter HIC.

Whereas the problem of stress cracking in a wet sour environment was identified as early as the $1950 \mathrm{~s}$, it was only d couple of decades later that hydrogen induced cracking as a mode of pipeline failure was appreciated (Moore \& Warga 1976). This is different from sulphide stress cracking (SSC) in that it can occur in stressed as well as unstressed bodies. Burns (1976) pointed out the difference in the appearance of these two types of sour environment cracks. HIC was identified as cracking due to a mechanism involving the formation, growth and linkage of internal blisters which often gave it the appearance of a series of steps and hence its alternate name - "stepwise cracking (SWC)". In this article I have used the terms HIC and SWC interchangeably, signifying the same phenomenon. Ikeda et al (1977) provided a schematic of the SWC process, which has been included here as figure 2 .

\section{Variables affecting HIC}

\subsection{Environmental variables}

The important environmental variables are $p \mathrm{H}$, chloride content, temperature, $\mathrm{H}_{2} \mathrm{~S}$ concentration, presence of dissolved oxygen and the exposure time. The findings of 

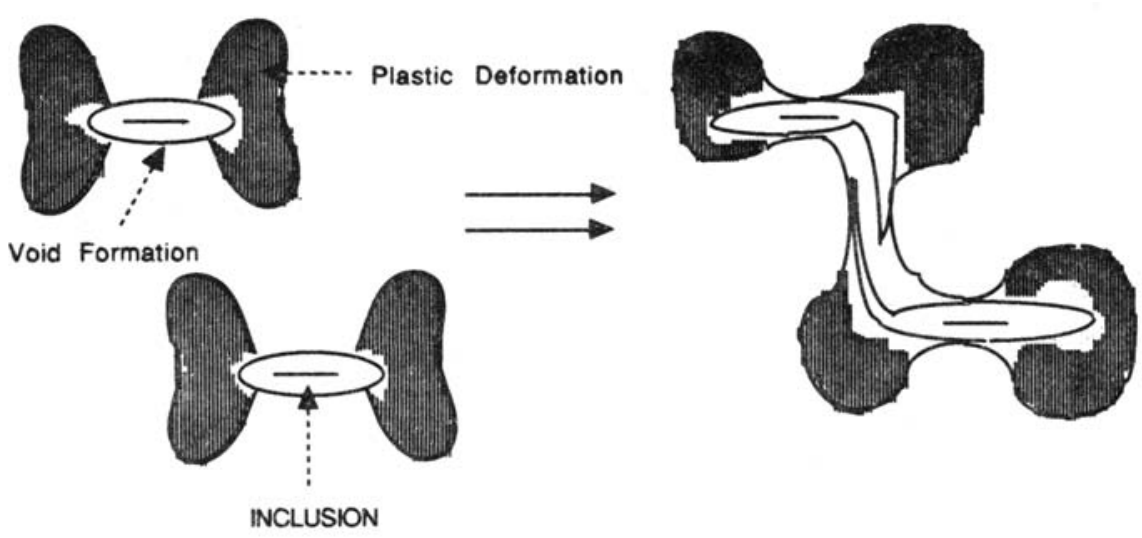

Figure 2 Direct linkage of two closely spaced cracks producing steps. After Ikeda et al (1977).

Ikeda et al (1980) indicated that lower $p \mathrm{H}$ environment accelerated corrosion as well as stepwise cracking (SWC). They also demonstrated that higher concentration of dissolved chlorides increased the severity of the environment. The extent of hydrogen damage was found (Kowaka et al 1975) to be maximum in the temperature range of 15 to $35^{\circ} \mathrm{C}$. Sharp reduction in SWC at temperatures above $35^{\circ} \mathrm{C}$ is attributed to the decreased concentration of $\mathrm{H}_{2} \mathrm{~S}$ in the saturated solutions. A threshold concentration of $\mathrm{H}_{2} \mathrm{~S}$ is often reported (Nakasugi et al 1979) below which HIC does not occur.

\subsection{Metallurgical variables}

One of the most important considerations is the casting practice with particular reference to the segregation of impurities and alloying elements occurring during casting. Moore \& Warga (1976) recommended the study of specimens from the pipe section of ingots (segregation zone), as these are most sensitive to SWC. They also found that the deoxidation practice during casting leads to increased SWC sensitivity so that fully killed steels are almost always more susceptible to HIC as compared :0 semi-killed steels of a similar composition. Moore $\&$ Warga pointed out that increased deoxidation increases sulphur solubility as well as promotes the formation of detrimental type II (stringers) MnS inclusions in comparison to the type I inclusions. Similarly, the practice of controlled rolling is conducive to the formation of $\mathrm{MnS}$ stringers. Coldren \& Tither (1976) concluded that SWC arises because of voids at non-metallic inclusion/steel interfaces. Wilde et al (1980) confirmed that SWC initiated at elongated type II MnS inclusions. Herbsleb et al (1980) also concluded that inclusions with sharp edges and large surfaces are preferred sites for initiation of SWC. After the realization that $\mathrm{MnS}$ inclusion shape and type are of vital importance in $\mathrm{HIC}$, various modifications in steelmaking practices have been tried out to control these inclusions. Decreasing the sulphur content was found beneficial by Herbsleb et al (1980) and by Brown $\&$ Jones (1984). The inclusion shape control by calcium or rare earth metal addition have been found to improve $\mathrm{HIC}$ resistance.

Miyoshi et al (1976) found that SWC propagated along anomalous microstructures composed of low temperature transformation products. Microstructural banding 
(pearlite or martensite) has also been found to increase crack propagation. The formation of banded microstructures in pipeline steels is due to dendritic segregation of manganese and phosphorus. According to Taira et al (1984) restricting the segregation zone hardness to below $300 \mathrm{HV}$ is needed to eliminate $\mathrm{HIC}$, since hard "banded" structures of bainite or martensite are susceptible to hydrogen embrittlement as well as HIC. Thus homogenization of microstructures by quenching and tempering would also reduce $\mathrm{SSC}$.

\subsection{Stress variables}

A correlation has not yet been established between steel strength and SWC susceptibility. $\mathrm{HIC}$ has been reported for a wide range of tensile strengths ( 300 to $800 \mathrm{M} \mathrm{Pa}$ ). It seems that nonmetallic inclusions and anomalous microstructures are more important factors in determining HIC susceptibility as compared to the strength of the steel. The SWC mechanism involves segregation of hydrogen atoms to internal interfaces followed by decohesion at these interfaces possibly by hydrogen pressurization. The plastic regions generated at the blister crack tips are embrittled by hydrogen, and transverse cracks propagate through the embrittled regions to join parallel cracksthus giving the appearance of steps. SWC occurs not only in unstressed components, but also in steels under elastic tensile stresses. Very few studies have been conducted to investigate the effect of welding residual stresses on the occurrence of SWC. The study of Taira et al (1979) seems to suggest that there is no effect of residual stresses.

\subsection{Chemical variables}

The addition of copper has often been found (Inagaki et al 1978) beneficial to SWC resistance of linepipe steels. This was attributed to the formation of a black protective film of Mackinawite, $\mathrm{FeS}_{1-x}$, enriched with $\mathrm{Cu}$. This reduces the corrosion rate as well as the amount of absorbed hydrogen. Thus $\mathrm{Cu}$-alloying is beneficial only in environments where a protective film can form. Similarly $\mathrm{Cr}$ additions were also found to improve SWC resistance. Nishimura et al (1977), and Parrini \& Devito (1978) had observed the beneficial effects of $\mathrm{Cr}$. Ni was also found to greatly reduce hydrogen absorption from sour environments (Nishimura et al 1977). Researchers have found that $\mathrm{Co}, \mathrm{Bi}$ and $\mathrm{Rh}$ additions cause decrease in hydrogen absorption by steels from $\mathrm{H}_{2} \mathrm{~S}$ containing environments (lino et al 1979).

\section{Case studies}

\subsection{First reported failures}

Parades \& Mize (1954) were the first to report service failure due to stepwise cracking (SWC). This occurred in 1951-52 on a API-5LX grade 359 transmission linepipe, after several months of service. It was found that the imperfections necessary for blistering were abundant in the material. Delaminations and blistering were evident with some blisters observed to have initiated at the delaminations. The analysis of the gas in the blister was found to contain $94 \%$ hydrogen, $2 \%$ methane, with traces of carbon dioxide 
and carbon monoxide. Class (1963) reported the failure of a pipeline in sour service. These were made from killed steels, were longitudinally welded by resistance welding and were used in sour service. Analysis revealed manganese sulphide and silicate inclusions in the material. It was also observed that seamless pipes performed significantly better in service.

\subsection{Hydrogen damage in a failed pressure vessel}

McHenry et al (1982) reported their investigation on a pressure vessel rupture at an oil refinery resulted in the death of 17 people and extensive property damage. The vessel was a $18.8 \mathrm{~m}$ high steel cylinder used to strip hydrogen sulphide from a process stream of propane and butane. The vessel was observed to fracture circumferentially adjacent to a repair weld made to replace a section damaged earlier by hydrogen blisters and delaminations. McHenry et al (1982) observed five types of hydrogen damage.

Hydrogen blisters and delaminations were observed in the bottom part of the cylinder. Blisters and delaminations were often close enough to the surface to cause visible bulging. The material was found to be quite susceptible to hydrogen induced cracking. These delaminations were indirectly responsible for failure as they led to the replacement of a section of the vessel. The steel matrix between the interfacial cracks was found to fail by cleavage because the hydrostatic stresses generated by absorbed hydrogen at blisters, inhibit plastic deformation. In addition, hydrogen induced cold cracking was observed in the heat affected zone of the repair weld. However McHenry et al (1982) did not believe that these modes were primarily responsible for failure. According to them, hydrogen stress cracks developed during operation of the vessel at the inside surface adjacent to the repair welos and hydrogen pressure cracking caused these surface cracks to propagate in a zigzag fashion through the vessel wall. These were facilitated by hydrogen embrittlement of the material which reduced the fracture toughness of the material by more than half.

\subsection{Stepwise cracking in a spirally welded pipeline}

Moore \& Warga (1976) reported that in 1974, three service ruptures occurred in a spirally welded, API $5 \mathrm{LX}$-Grade 42 sour gas transmission pipeline between four to seven weeks after commissioning. It was found that extensive blistering and cracking had occurred over $10 \mathrm{~km}$ of this $90 \mathrm{~km}$ pipeline. The failures could be attributed to stepwise cracking near elongated $\mathrm{MnS}$ inclusions. Although the failures occurred near the spiral weld, they could not be associated with any kind of weld defects. Moore \& Warga used a test proposed by Cotton to study the HIC susceptibility of the material. They found that the most important factor affecting the hydrogen cracking sensitivity of steels was the $\mathrm{MnS}$ inclusion morphology. Elongated type II, MnS stringers were observed to render the steel very crack sensitive. Moreover, since all fully killed steels except those treated with rare earth elements contain type II MnS inclusions, they would be more susceptible to HIC than semi-killed steels. Moore \& Warga were of the opinion that although steels can be made insensitive to HIC, a completely immune steel was not feasible. 


\subsection{Ongoing research at the National Metallurgical Laboratory, Jamshedpur}

Under the aegis of The Component Integrity Evaluation Programme (CIEP), which is a concerted effort on life estimation of various components, one of the projects is focusing on the studies of HIC in API 5 LX Grade 52 pipeline steel exposed to simulated sour environment. This investigation is an attempt at an 'in toto' observation of the mechanical property degradation brought about by sour environment exposure, and is one of the few attempts at correlating the extent of HIC with the loss in ductility of the material. One of the observations of this study was that the extent of HIC was a monotonically increasing function of the time of exposure to the sour environment. It was found that even after twenty-eight days of exposure to the NACE prescribed sour environment, no saturation of the HIC damage occurred. This observation indicated the great potential of lattice imperfections to trap hydrogen. In this investigation the mechanical property deterioration caused by HIC was studied in some detail through slow straining after exposure to the sour environments. The investigators found that, surprisingly, hydrogen induced cracks, per se, did not cause a deterioration of ductility. Such deterioration was the result of the presence of mobile lattice hydrogen which are distinct from hydrogen trapped at interfaces of matrix/ inclusions, the latter being responsible for HIC. This investigation has so far revealed the clearly distinct roles of mobile lattice hydrogen and trapped hydrogen, each causing different types of damage to the matrix, the former causing lattice embrittlement most observable on dynamic straining and the latter causing cracking under static conditions.

\section{Preventive measures}

The preventive measure which can be adopted during service include the injection of inhibitors, desulphurization and dehydration of the gas passing through the line. National Association of Corrosion Engineers (NACE) recommended a hardness limit of HRC 22 for steels in use in sour environments. Various metallurgical parameters can also be controlled to reduce HIC.

There are three well-known measures to improve the HIC resistance.

(1) reduction of hydrogen entering into steels by the formation of protective films.

(2) reduction of $\mathrm{MnS}$ and inclusion shape control.

(3) control of microstructures, especially segregation zones.

The first of these methods is often insufficient as the film formation is $p \mathrm{H}$ dependent and in aggressive media (low $p \mathrm{H}$ ) protective films are often not formed. It was argued earlier that the beneficial effect of $\mathrm{Cu}$ alloying was due to the enrichment of the protective surface film of makinawite. The more effective preventive measures are the modification of inclusion shape and amount, and the control of the microstructure.

Taira et al (1984) found that complete elimination of inclusion improves SSC resistance as well as $\mathrm{HIC}$ resistance. It was mentioned earlier that fully killed steels were more prone to HIC, however, due to their better mechanical properties, it is often desirable to use them in spite of the HIC limitation. This calls for modifications in the steelmaking practice. Sulphide shape control by rare earth additions is often used. However since the amount of rare earth addition has to be very precise, steelmakers are often tentative about following this practice. Moreover, rare earth sulphides tend to 
produce ionization effects which interfere with arc stability in automatic welding processes. Calcium treated steels were also found to have improved resistance to HIC (Brown \& Jones 1984; Taira et al 1984). According to Taira et al (1984) calcium content must be delicately controlled so that neither manganese sulphide, nor calcium sulphide clusters are present. They believed that the HIC resistance of a calcium-treated steel depended on the ratio of the Ca content to the S content. Brown \& Jones (1984) found that $\mathrm{HIC}$ resistance increased with $\mathrm{Ca}: \mathrm{S}$ ratio. They observed that rare earth metal treated steels were less resistant than Ca-treated steels.

HIC is often found to propagate along microstructural bands (pearlitic). The formation of the latter is a result of dendritic segregation of $\mathrm{Mn}$ and $\mathrm{P}$. The hardness of the centre segregation in steel plates is one of the most important factors affecting HIC susceptibility of high strength line pipe steels. Matsumoto et al (1986) found that lowering the carbon content to below $0.05 \%$ decreased the hardness of the segregation zones. They also found that homogenization treatments were effective in decreasing the same. However such treatments are time consuming due to the slow diffusion of $\mathrm{Mn}$ and may not be practical, causing reduced industrial productivity. Brown \& Jones (1984) advocated the use of quenched and tempered steels and reduction of manganese to reduce banding. They also suggest the use of modern casting techniques like superheat temperature control and electromagnetic stirring to reduce the centreline segregation in continuously cast steels.

\section{References}

Bastien P, Azou P 1951 Influence de l' amplitude vitesse des deformations plastiques sur la segretation de la hydrogene dans le fer et les aciers. Compte. Rendus 232: 69-71

Beck W, Bockris J O'M. McBreen J, Nanis L 1965 Hydrogen permeation in metals as a function of stress temperature and dissolved hydrogen concentration. Proc. R. Soc. A290: $220-235$

Bilby B A, Hewitt J W 1962 Hydrogen in steels - the stability of microcracks. Acta Metall. 10: $587-600$

Birnbaum H K, Mazzolai F M 1985 Elastic constants and ultrasonic attenuation of the alpha-alpha prime phase of the $\mathrm{Nb}-\mathrm{H}$ (D) system. J. Phys. F15: $507-542$

Bockris J O'M, Subramanyan P K 1971 The equivalent pressure of molecular hydrogen in cavities within metals in terms of the overpotential developed during the evolution of hydrogen. Electrochim. Acta 16:2160 2179

Briant C L, Messmer R P 1980 Electronic effects of sulphur in nickel - A model for grain boundary embrittlement. Philos. Mag. 42: 569-576

Brown A, Jones C L 1984 Hydrogen induced cracking in pipeline steels. Corrosion 40:330-336

Burns D S 1986 Laboratory tests for evaluating alloys for hydrogen sulphide service. Mat. Perf. 15: 21 - 28

Class 11963 Report on investigation of sulphide stress corrosion cracking of steels, particularly of steels of comparatively low tensile strength. 2nd Int. Conf. Met. Corr., New York

Coldren A P. Tither G J 1976 Mctallographic study of hydrogen induced cracking in line pipe steel. J. Metall. $28(5): 5 \cdot 10$

Darken L S, Smith R P 1949 Behaviour of hydrogen in steel during and after immersion in acid. Corrosion 5: 1-16

de Kazinczy F 1954 A theory of hydrogen embrittlement. J. Iron. Steel Inst. 177: $85 \cdots 92$

Garofalo F, Chetu Y T, Ambegackar V 1960 Effect of hydrogen on stability of microcracks in iron and steels. Acta Metall. 8: $504 \div 512$

Hancock G G, Johnson H H 1965 Hydrogen oxygen and subcritical crack growth in a high strength stee!. Trans. Metall. Soc. AIME 236: 513-516 
Herbsleb G, Popperling R K, Schwenk W 1980 Occurrence and prevention of hydrogen induced stepwsie cracking and stress corrosion cracking of low alloy pipeline steels. NACE Natl. Conf., Chicago, NACE

Iino M, Nomura N, Takezawa H, Gondoh H 1979 Aciers pour pipe-lines resistant au cloquage et au criquage dus a l' hydrogene. Rev. Metall. 76: 591

Ikeda A, Morita Y, Terasaki F, Takeyama M 1977 On the hydrogen induced cracking of line-pipe steel under wet hydrogen sulphide environment. 2nd Int. Cong. on hydrogen in metals, Paris Paper no. 4A7

Ikeda A, Terasaki F, Kaneko T 1980 Influence of environment conditions and metallurgical factors on hydrogen induced cracking of line pipe steel. NACE Natl. Conf. Chicago

Inagaki H, Tanimura M, Matsushima L, Nishimura T 1978 Effect of $\mathrm{Cu}$ on the hydrogen induced cracking of pipeline steel. Trans. Iron Steel Inst. Jpn. 18: 149-156

Johnson H H, Troiano A R 1957 Crack initiation in hydrogenated steels. Nature (London) 179: 777

Johnson H H, Hirth J P 1976 Internal hydrogen supersaturation produced by dislocation transport. Metall. Trans. A7: 1543-1548

Johnson H H, Quick N, Kumnick A J 1979 Hydrogen trapping mechanisms by permeation techniques. Scr. Met. 13: 67-72

Kowaka M, Terasaki F, Nagata S, Ikeda A 1975 The test method of hydrogen induced cracking of rolled steels under wet hydrogen sulfide environment. The Sumitomo Search 14(11): 36

Matsumoto K, Kobayashi Y, Ume K, Murakami K, Taira K, Arikata K 1986 Hydrogen induced cracking susceptibility of high strength linepipe steels. Corrosion 42: 337-345

McBreen J, Genshaw M A 1967 Proc. Intl. Symp. on SSC, NACE Houston

McHenry H I, Purtscher P T, Shives T R 1982 Observations of hydrogen damage in a failed pressure vessel. Corrosion Sci. 27: 1041-1057

McNabb A, Foster P K 1972 A new analysis of the diffusion of hydrogen in iron and ferritic steels. Trans Metall. Soc. AIME 227: 618-627

Miyoshi E, Tanaka T, Terasaki F, lkeda A 1976 A hydrogen induced cracking of steels under wet hydrogen sulphide environment. Trans. ASME, J. Eng. Ind. B98: 1221

Moore E M, Warga J J 1976 Factors influencing the hydrogen cracking sensitivity of pipeline steels. Mater. Perform. 15(6): 17-23

Mortlet J G, Johnson H H, Troiano A R 1958 A new concept of hydrogen embrittlement in steels. J. Iron. Steel Inst. 189: 37-44

Nakasugi H, Sugimura S, Matsuda H, Murata T 1979 Development of new linepipe steels for sour gas service. Nippon Steel Tech. Report 14(12): 66

Nishimura T, Inagaki H, Tanimura M 1977 Hydrogen cracking in sour gas pipeline steel. 2nd Intl. Cong. on hydrogen in metals, Paris, Paper No. 3E9

Oriani R A, Josephic P H 1974 Equilibrium aspects of hydrogen induced cracking of steeis. Acta Metall. 22: 1065-1074

Oriani R A, Josephic P H 1979 Hydrogen enhanced load relaxation in a deformed medium carbon steel. Acta Metall. 27: 997-1005

Oriani R A, Josephic P H 1980 Effect of hydrogen on the plastic properties of medium carbon steels. Metall. Trans. A11: 1809-1820

Parades F, Mize W W 1954 Oil Gas J. 53: 99

Parrini C, Devito A 1978 High strength microalloyed pipe steels resistant to hydrogen induced failures. Proc. MICON'78, Houston, (Ohio: ASM)

Pressouyre G M, Bernstein I M 1978 A quantitative analysis of hydrogen trapping. Metall. Trans. A9: 1571-1580

Robertson I M, Tabata T, Wei W, Heubaum F, Birnbaum H K 1984 Hydrogen embrittlement and grain boundary fracture. Scr. Metall. 18: 841-846

Smialowski M 1962 Hydrogen in steels (London: Pergamon)

Taira T, Kobayashi Y, Inagaki H, Watanabe T 1979 Sulphide corrosion cracking of line-pipe for sour service. NACE Natl. Conf. Atlanta 1979

Taira T, Kobayashi Y, Matsumoto K, Tsukada K 1984 Resistance of line pipe steels to wet sour gas. Corrosion 40: 478-486

Tetelman A S, Robertson W D 1963 Direct observations and analysis of crack propagation in iron $-3 \%$ silicon single crystals. Acta Metall. 11: $415-426$ 
Troiano A R 1960 The role of hydrogen and other interstitials in the mechanical behaviour of metals. Trans. Am. Soc. Metall. 52: 54-80

Wilde B E, Kim C D, Phelps E H 1980 Some observations on the role of inclusions in hydrogen induced blister cracking of linepipe steels in sulphide environments. NACE Natl. Conf., inici: $\mathrm{g}_{1}$

Zapfle C A, Sims C E 1941 Hydrogen embrittlement internal stress and defects in steels. Trans. Am. Inst. Min. Metall. Eng. 145: 225.259 